\title{
Gastrointestinal Mucormycosis: A Rare but Lethal Mimicker of Necrotising Enterocolitis
}

\author{
Veerabhadra R ${ }^{1}$, Krishnakumar $\mathbf{G}^{2}$, Bharathi $\mathrm{B}^{3}$, Nishad $\mathrm{P}^{4}$, Sree Rekha $\mathrm{J}^{5}$
}

${ }^{1}$ Dr. Veerbhadra Radhakrishna, MBBS, MS, MCh. Department of Paediatric Surgery, ${ }^{2} \mathrm{Dr}$. Krishna Kumar Govindarajan, MBBS, MS, MCh. Department of Paediatric Surgery, ${ }^{3} \mathrm{Dr}$. Bharathi Balachandar, MBBS, MD, DM. Department of Neonatology, ${ }^{4}$ Dr. Nishad Plakkal, MBBS, MD. Department of Neonatology, ${ }^{5} \mathrm{Dr}$. Sree Rekha Jinkala, MBBS, MD. Department of Pathology. All from the Jawaharlal Institute of Postgraduate Medical Education and Research, Pondicherry, India.

\section{Address for correspondence:}

Dr. Krishna Kumar Govindarajan

E-mail:kkpeds@gmail.com

\section{How to cite}

Veerabhadra R, Krishnakumar G, Bharathi B, Nishad P, Sree Rekha J. Gastrointestinal Mucormycosis: A Rare but Lethal Mimicker of Necrotising Enterocolitis. J Nepal Paediatr Soc 2016;36(2):198-200.

doi: http://dx.doi.org/10.3126/jnps.v36i2.15955

This work is licensed under a Creative Commons Attribution 3.0 License.

\section{(c) (P)}

\begin{abstract}
Neonatal Gastrointestinal mucormycosis is a rare fungal infection carrying high morbidity and mortality. Clinically, it is not easily distinguishable from the more common necrotizing enterocolitis. Diagnosis is made by histopathology of the involved bowel. We report a neonate with gastrointestinal mucormycosis, bringing to notice the fatality of this infection.
\end{abstract}

Key words: Gastrointestinal mucormycosis, Necrotizing enterocolitis, neonatal bowel perforation

\section{Introduction}

M ucormycosis is an opportunistic fungal infection seen classically in patients with diabetes, lymphoma, leukemia, burns, malignancy, steroid usage, renal impairment on peritoneal dialysis and immunomodulatory usage due to organ transplant ${ }^{1}$. Neonatal mucormycosis is a rare infection which is difficult to diagnose and hence carries a high mortality ${ }^{2}$. Here we report a case of neonatal gastrointestinal mucormycosis, outlining the management and diagnostic dilemma, which the case presented.

\section{The Case}

An extramural, 33 weeks, baby girl, $1^{\text {st }}$ of triplets, presented with sudden onset respiratory distress and lethargy from second day of life. After admission and stabilization in the neonatal intensive care unit, she was started on broad spectrum intravenous antibiotics and tube feeds. She developed distension of abdomen on the fifth day of life. Plain x-ray of abdomen showed non-specific bowel dilatation without evidence of pneumatosis or pneumoperitoneum.

With a provisional diagnosis of necrotizing enterocolitis, conservative management was planned with nil per oral and intravenous fluids. Subsequently, the abdominal distension was progressive and repeat $x$-ray of abdomen was found to have hollow viscus perforation on day 13 of life (Fig 1). With this, the child was referred to us for further management. At presentation, her activity was poor, capillary filling time was more than three seconds, heart rate was $164 / \mathrm{min}$, respiratory rate was $35 / \mathrm{min}$. Systemic examination including cardiovascular and respiratory system examination was normal. On palpation, abdomen was tense and tender. Laboratory parameters were $\mathrm{Hb} 12.3 \mathrm{~g} / \mathrm{dl}$; total white cell count was $7150 / \mathrm{mm}^{3}$ 
with neutrophils $81 \%$ and platelet count 1.56 lakhs/ $\mathrm{mm}^{3}$. She was diagnosed as Necrotizing Enterocolitis stage IIIb and was planned for emergency surgery after resuscitation. Informed written consent for exploratory laparotomy was obtained from parents.

Intra-operatively, about $40 \mathrm{ml}$ of feculent peritoneal fluid was present. Distal half of transverse colon and descending colon was found to be gangrenous. Unhealthy colon was resected and peritoneal lavage was given. The proximal part of right transverse colon was brought out as end stoma with the healthy sigmoid colon as mucus fistula. Post operatively, the child was ventilated. Her vitals were unstable in spite of inotropic support and close monitoring in the neonatal intensive care unit. Finally, she succumbed to multi-organ dysfunction on $2^{\text {nd }}$ post-operative day. Intra-operative pus grew pan-resistant Enterococcus faecalis and Candida.

Histopathology of resected segments revealed extensive ulceration of bowel with serositis. Broad fungal elements were seen with angio-invasion at places. Features were consistent with gastrointestinal mucormycosis (Figure 2 and 3 ).

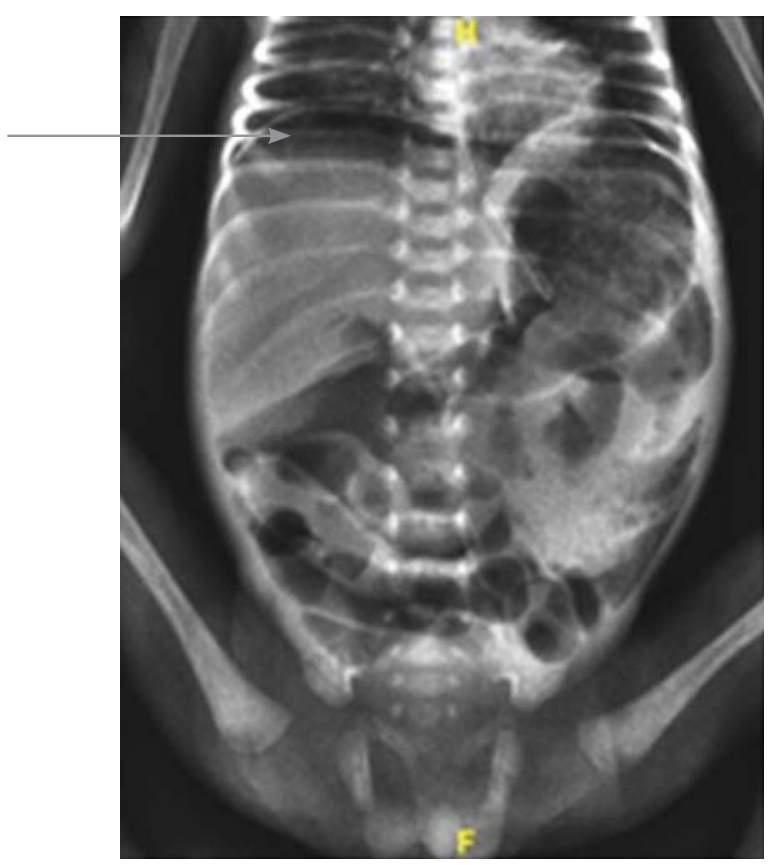

Fig 1: X-ray abdomen showing pneumoperitoneum

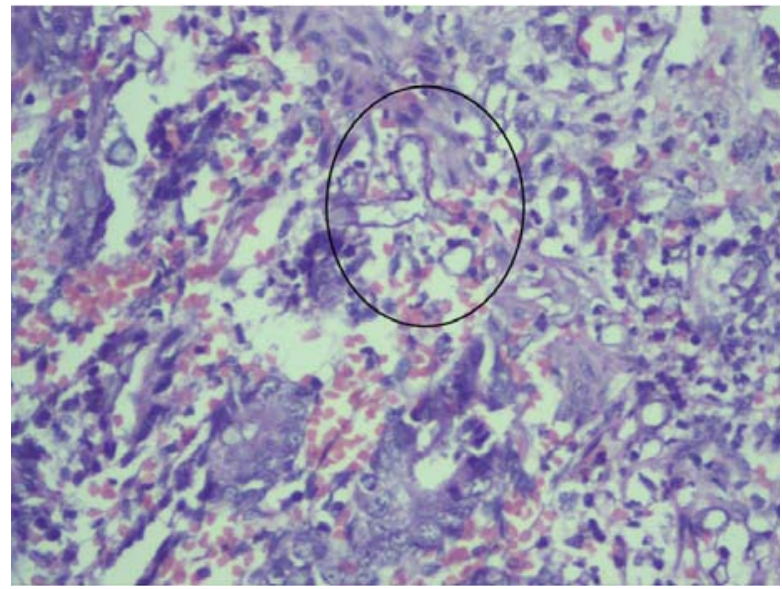

Fig 2: Section from colon showing few colonic glands and broad aseptate basophilic fungal profiles of mucormycosis at the muscularis mucosae $\mathrm{H} \& \mathrm{E} X 200$

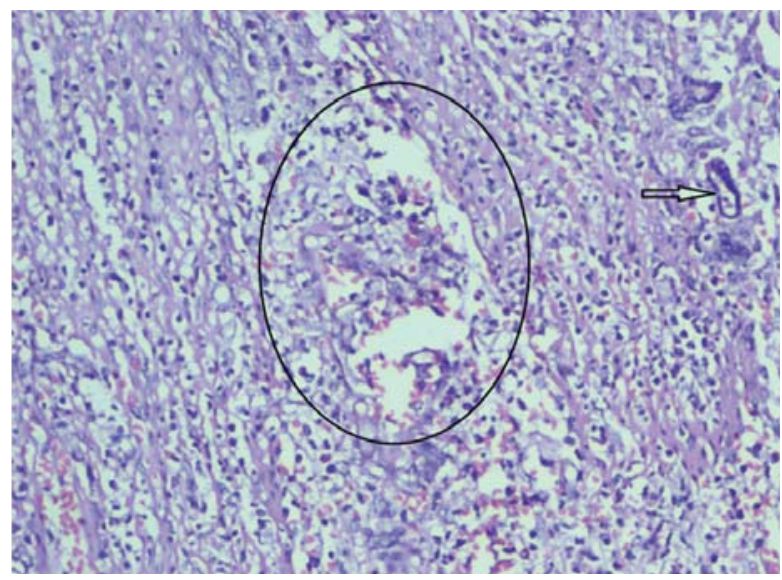

Fig 3: Muscularispropria showing broad aseptate basophilic fungal profiles of mucormycosiswith a gaint cell reaction (arrow) H \& E X200

\section{Discussion}

Zygomycosis is a rare, life-threatening fungal infection caused by Zygomycetes seen in immunocompromised hosts. Mucor, Rhizomucor and Absidia are the zygomycetes affecting humans. These are ubiquitous saprophytes, found in the soil and decomposing organic substance. The airborne spores formed by these fungi are infective which when inhaled, ingested or injected leads to cerebral, respiratory, gastrointestinal or cutaneous infections..$^{1,2}$

Gastrointestinal mucormycosis is the rarest form of zygomycosis constituting $7 \%$ of all cases. Till date around 60 cases have been reported with only $25-30 \%$ survival rate. Stomach is the most commonly affected organ in children and adults while colon is the most commonly affected organ in neonates ${ }^{3,4}$. 
Premature and low birth weight (LBW) neonates are at high risk as the immune system is immature and skin is fragile making them highly susceptible. Approximately $83 \%$ of gastrointestinal mucormycosis occurs in preterm neonates ${ }^{5}$. The humid neonatal intensive care unit environment enhances the growth of these fungi. Broad spectrum antibiotics and steroids affect healthy gastrointestinal flora whereas naso or oro-gastric tube, endotracheal tube and indomethacin damage the gut increasing the risk of Mucor invasion ${ }^{3,4}$. Co-existing congenital heart disease with splanchnic hypoperfusion, nil oral status with avoidance of breast milk, acidosis and low gastric $\mathrm{pH}(\leq 6.5)$ also carry a high risk for mucormycosis ${ }^{5}$. Our patient had most of the risk factors.

It's difficult to differentiate gastrointestinal mucormycosis from necrotizing enterocolitis (NEC) in view of their similar clinical presentation. Neutropenia, absence of pneumatosis intestinalis and poor response to broad-spectrum antibiotics can give a clue towards Mucormycosis, but all these are non-specific features ${ }^{3,4}$. Fungal cultures are positive in only $33 \%$ cases. ${ }^{5}$ Colonic involvement with sparing of rest of the small bowel, as in our case, points towards Mucormycosis. ${ }^{4}$ As these fungi have an affinity towards arterial involvement, the resultant arterial thrombosis leads to extensive ischaemic necrosis of the bowel which is out of proportion to the degree of infection. ${ }^{5}$ Widespread angio-invasion and presence of fungal hyphae in histopathology is diagnostic of Mucormycosis $3,4,5$.

Early, aggressive and adequate surgical resection followed by intravenous Amphotericin- B is the mainstay of treatment. Antifungal agents cannot reach the infected tissue as the agent is blocked by the presence of mycotic thrombi3 ${ }^{3,5}$. Adequate resection reduces the fungal load and improves the efficacy of antifungal therapy. Adequate resection also reduces the risk of perforation and stricture formation. Primary anastomosis is undesirable while there are reports of extensive gangrene of abdominal wall post-stoma ${ }^{5}$. Hence clip and drop, keeping laparotomy incision as laparostomy to inspect tissues is recommended by a few authors ${ }^{5}$. This is followed by delayed primary anastomosis, once general condition improves. It is of note that the commonly used antifungal agent, fluconazole and even newer agents like caspofungin and voriconazole are not effective against Mucormycosis. ${ }^{3}$

The prognosis of gastrointestinal Mucormycosis is grim with high mortality. Poor outcome is due to lack of clinical suspicion, inadequate surgery and ineffective antifungal therapy. The survival depends on the virulence of the organism, timely intervention and immune status of the individual. Though our patient underwent adequate surgical resection, adequate antifungal therapy was not initiated. In addition, contributing factor for our patient's mortality was also the late presentation with bowel perforation.

\section{Conclusion}

A high index of suspicion of gastrointestinal mucormycosis is a must in preterm and low birth weight neonates with clinical features of necrotizing enterocolitis having neutropenia. Early aggressive adequate surgery followed by commencement of amphotericin-B is the mainstay of treatment. We suggest frozen section as a modality to quicken diagnosis and initiate appropriate treatment.

\section{References}

1. Spellberg B. Gastrointestinal mucormycosis: An evolving disease. GastroenterolHepatol 2012;8(2):140-2.

2. Sridhar S., Atanu K J, Susanna T and Rekha S. Mucormycosis of the Neonatal Gastrointestinal tract. Indian Pediatr 2001;38:294-7.

3. Sarin YK. Intestinal mucormycosis in a neonate: A case report and review. J Indian AssocPediatrSurg 2010;15(3):98-100.
4. Patra S, Vij M, Chirla DK, Kumar N, Samal SC.. Unsuspected invasive neonatal gastrointestinal mucormycosis: A clinicopathological study of six cases from a tertiary care hospital. J Indian AssocPediatrSurg 2012;17(4):153-6.

5. Raveenthiran V. Gastrointestinal Mucormycosis Mimicking Necrotizing Enterocolitis of Newborn. J Neonatal Surg 2013;2(4):41-4. 